Article

\title{
The Histone Deacetylase Inhibitor Suberoylanilide Hydroxamic Acid (SAHA) Restores Cardiomyocyte Contractility in a Rat Model of Early Diabetes
}

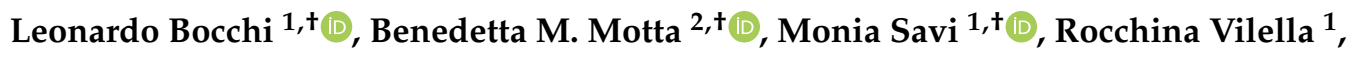 \\ Viviana Meraviglia ${ }^{2}$, Federica Rizzi ${ }^{3}$ (D), Serena Galati ${ }^{1}$, Annamaria Buschini ${ }^{1}$ (D), \\ Mirca Lazzaretti ${ }^{1}$, Peter P. Pramstaller ${ }^{2}$, Alessandra Rossini ${ }^{2, * \mathbb{D}}$ and Donatella Stilli ${ }^{1, *}$ \\ 1 Department of Chemistry, Life Sciences and Environmental Sustainability, University of Parma, \\ 43124 Parma, Italy; leonardo.bocchi@unipr.it (L.B.); monia.savi@unipr.it (M.S.); \\ rocchina.vilella@studenti.unipr.it (R.V.); serena.galati@unipr.it (S.G.); annamaria.buschini@unipr.it (A.B.); \\ mirca.lazzaretti@unipr.it (M.L.) \\ 2 Institute for Biomedicine, Eurac Research, 39100 Bolzano, Italy \\ (affiliated institute of the University of Lübeck, 23562 Lübeck, Germany); \\ Benedetta.Motta@eurac.edu (B.M.M.); viviana.meraviglia@gmail.com (V.M.); \\ Peter.Pramstaller@eurac.edu (P.P.P.) \\ 3 Department of Medicine and Surgery, University of Parma, 43126 Parma, Italy; federica.rizzi@unipr.it \\ * Correspondence: Alessandra.Rossini@eurac.edu (A.R.); donatella.stilli@unipr.it (D.S.); \\ Tel.: +39-0471-055504 (A.R.); +39-0521-906117 (D.S.) \\ + These authors contributed equally to this work.
}

Received: 16 March 2019; Accepted: 12 April 2019; Published: 16 April 2019

\begin{abstract}
In early diabetes, hyperglycemia and the associated metabolic dysregulation promote early changes in the functional properties of cardiomyocytes, progressively leading to the appearance of the diabetic cardiomyopathy phenotype. Recently, the interplay between histone acetyltransferases (HAT) and histone deacetylases (HDAC) has emerged as a crucial factor in the development of cardiac disorders. The present study evaluates whether HDAC inhibition can prevent the development of cardiomyocyte contractile dysfunction induced by a short period of hyperglycemia, with focus on the potential underlying mechanisms. Cell contractility and calcium dynamics were measured in unloaded ventricular myocytes isolated from the heart of control and diabetic rats. Cardiomyocytes were either untreated or exposed to the pan-HDAC inhibitor suberoylanilide hydroxamic acid (SAHA) for $90 \mathrm{~min}$. Then, a fraction of each group of cells was used to evaluate the expression levels of proteins involved in the excitation-contraction coupling, and the cardiomyocyte metabolic activity, ATP content, and reactive oxygen species levels. SAHA treatment was able to counteract the initial functional derangement in cardiomyocytes by reducing cell oxidative damage. These findings suggest that early HDAC inhibition could be a promising adjuvant approach for preventing diabetes-induced cardiomyocyte oxidative damage, which triggers the pro-inflammatory signal cascade, mitochondrial damage, and ventricular dysfunction.
\end{abstract}

Keywords: diabetes; HDAC inhibition; cardiomyocyte mechanics; calcium transients; cell oxidative stress

\section{Introduction}

Diabetes is a risk factor for the development of various cardiovascular complications, which constitute the leading causes of morbidity and mortality in both type 1 and type 2 diabetic subjects [1]. The incidence of cardiovascular diseases is common in diabetic patients and accounts for 
$80 \%$ of deaths, even in the presence of a tight control over metabolic state and blood glucose levels by existing therapeutic agents. Despite extensive investigation, development of effective treatments against the complications of diabetes has proven challenging [2,3].

Hyperglycemia is a central player in activating several adaptive and maladaptive responses in myocardial tissue among which cell oxidative stress and moderate tissue inflammation constitute critical early pathogenic components [4-9]. Mitochondrial injury also has a causative role in the pathophysiology of diabetic heart disease $[5,10]$, largely contributing to the generation of reactive oxygen species (ROS) and myocardial inflammation, leading to the development and progression of cardiac abnormalities and dysfunction. Hence, there is a need to identify novel adjuvant therapeutic approaches specifically aimed at counteracting the initial hyperglycemia-induced alterations in myocardial tissue.

In this context, histone acetyltransferases (HAT) and histone deacetylases (HDAC) have recently gained great attention as important molecules involved in the regulation of a variety of cellular responses, as well as in the modulation of pathological conditions, including diabetes and heart failure [11-14]. Histone acetylation mediated by HAT results in the modification of the structure of chromatin, leading to nucleosome relaxation and transcriptional activation. It follows that the acetylation of nucleosome histone tails provides a critical mechanism for epigenetic control of gene expression. In contrast, the reverse reaction mediated by HDACs induces de-acetylation, chromatin condensation, and transcriptional repression [15]. The interplay between HATs and HDACs, which physiologically determinates the acetylation status of histone tails, recently has emerged as a crucial factor in the development of cardiac disorders with different etiologies [2]. Additionally, proteomic studies have revealed that non-histone proteins are also subjected to reversible lysine acetylation [13], further highlighting the biological significance of this post-translational modification.

In the setting of diabetes, recent evidence indicates that HDAC inhibition increases the levels of antioxidant enzymes and reduces cardiac dysfunction in experimental models of advanced type I diabetes [16] and ameliorates cardiac performance and metabolic disturbances in murine models of both type I and type II diabetes [2,17]. An additional study [18] shows that chronic in vivo treatment of diabetic mice with a HDAC3 inhibitor, besides preventing diabetes-induced ventricular dysfunction and remodeling, maintains cardio-protective effects for at least three months after the end of treatment, like a "protective memory" phenomenon. Furthermore, Wu et al. [19] recently documented that HDAC inhibition attenuates ischemia/reperfusion cardiac injury in diabetic hearts.

Previous data, although strongly suggestive that HDAC inhibitors may constitute a potential new therapeutic tool for the treatment of diabetic cardiomyopathy, mainly are concerned with the advanced stages of diabetes. On the other hand, we previously showed that alterations in the redox state of cardiac cells and the activation of pro-inflammatory signal cascades occur from the initial stages of diabetic disease [4], resulting in the first signs of impaired cardiomyocyte contractility and calcium dynamics, well before the appearance of the overt cardiomyopathy phenotype and collagen accumulation in cardiac tissue $[7,20,21]$.

To the best of our knowledge, the cardioprotective effects of HDAC inhibition at the very early stages of diabetes and the analysis of the potential underlying mechanisms have never been explored. To specifically address this issue, we evaluated the functional and metabolic response to HDAC inhibition of cardiomyocytes (CMs) isolated from rats with streptozotocin (STZ) induced diabetes after three weeks of hyperglycemia. The small molecule suberoylanilide hydroxamic acid (SAHA), a pan-HDACs inhibitor [15], was used. Notably, SAHA (Vorinostat) use was approved by the US FDA in October 2006 for the treatment of refractory cutaneous T-cell lymphoma [22]. Recently, we reported that SAHA-induced HDAC inhibition ameliorates intracellular $\mathrm{Ca}^{2+}$ dynamics and, accordingly, contractile performance in normal adult rat $\mathrm{CMs}$, supporting the hypothesis that the modulation of the HAT/HDAC interplay by small active molecules can positively affect the functional properties of the CMs [23]. 
We found here that SAHA-induced HDAC inhibition was able to prevent the occurrence of the first signs of cardiomyocyte functional derangement in the diabetic heart mainly by reducing the accumulation of intracellular ROS. These findings may open new ways for the development of therapeutic approaches based on small molecules capable of counteracting the initial functional damage induced by diabetes in myocardial tissue.

\section{Results}

\subsection{Effect of SAHA Treatment on Cardiomyocyte Mechanics and Calcium Transients}

Ex-vivo experiments were performed to evaluate the ability of SAHA exposure to ameliorate calcium dynamics and contractile properties of CMs isolated from adult rats after a short period of hyperglycemia ( 3 weeks) that constitutes a time point characterized by the occurrence of the first signs of dysfunction, as measured at the cellular level [4]. Diabetic CMs were either untreated (D group) or incubated with $2.5 \mu \mathrm{mol}$ SAHA (D + SAHA group) for $90 \mathrm{~min}$.

The average diastolic sarcomere length was approximately equal to $1.7 \mu \mathrm{m}$ in both control (C group) and diabetic CMs, independent of the treatment. Conversely, in accordance with previous data [4], intracellular $\mathrm{Ca}^{2+}$ handling and contraction/relaxation properties measured in unloaded ventricular myocytes isolated from D hearts were partially impaired compared to C (Figure 1). Slower calcium kinetics were observed in D cells, which exhibited a prolonged time-to-peak of the calcium transient (TTP, $+19 \%, p<0.01$, Figure 1D) associated with higher values of the time required for cytosolic calcium clearing (tau, $+26 \%, p<0.01$, Figure $1 \mathrm{E}$ ), in the absence of statistically significant differences in the amplitude of the calcium transient ( $\mathrm{F} / \mathrm{F} 0$, fold increase) that showed only a slight decrease $(-9 \%$, Figure $1 \mathrm{C})$.

In line with the compromised calcium dynamics, the contractile efficiency of untreated D cells was significantly reduced, as documented by the decrease in the maximal rate of shortening $\left(-\mathrm{dL} / \mathrm{dt}_{\max }\right.$, $-25 \%$; Figure $1 \mathrm{G})$ and re-lengthening $\left(+\mathrm{dL} / \mathrm{dt}_{\max },-22 \%\right.$; Figure $\left.1 \mathrm{H}\right)$, leading to a global prolongation of re-lengthening times, measured at $50 \%$ and $90 \%$ of re-lengthening (RL50\% and RL90\%, Figure 1I,J). Conversely, the fraction of shortening exhibited only a moderate decline (FS, Figure 1F). SAHA exposure was able to induce a partial or complete recovery of most functional parameters that attained values comparable to those measured in C cells (Figure 1D-J). 

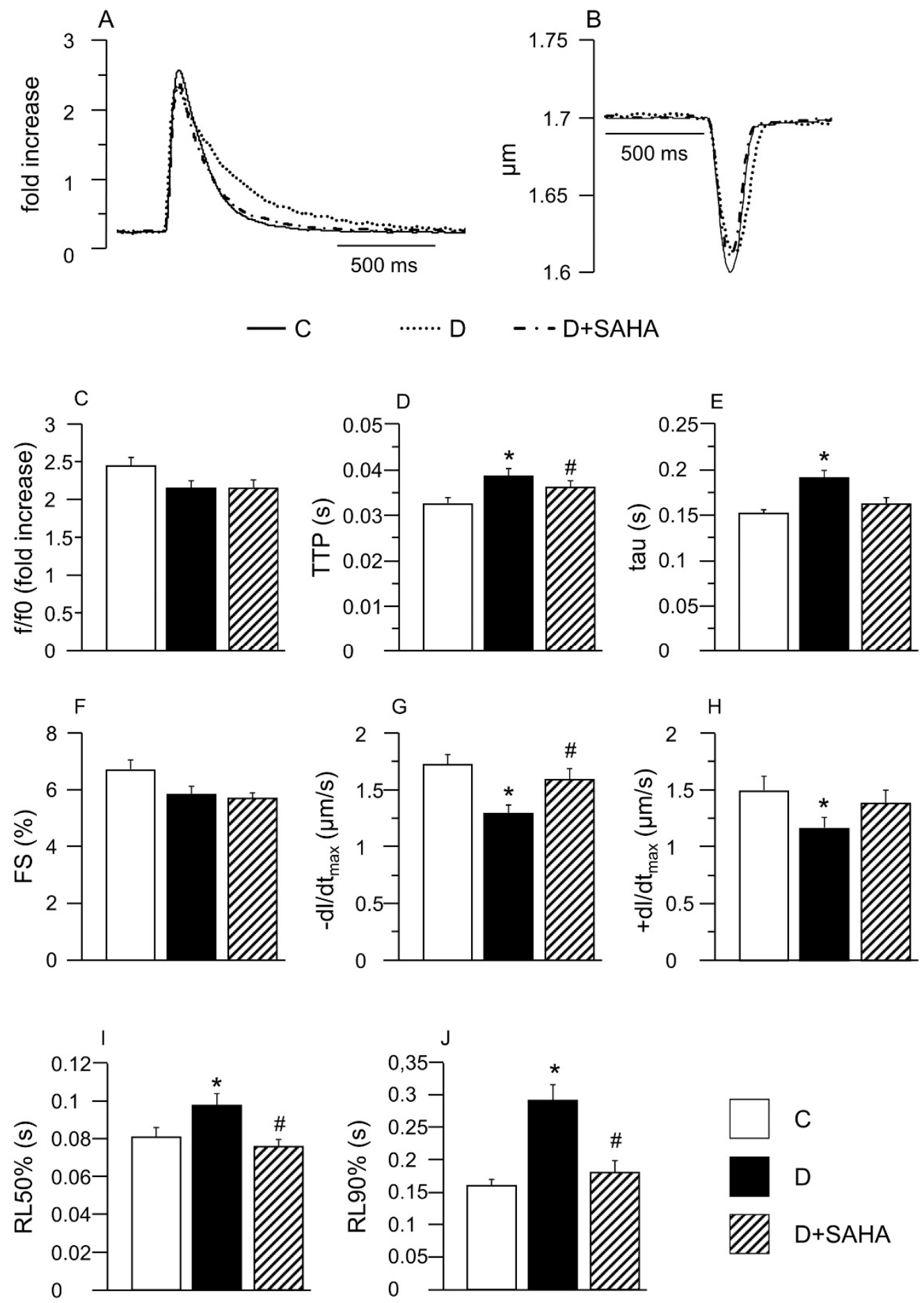

Figure 1. Effect of suberoylanilide hydroxamic acid (SAHA) treatment on calcium transients and cell mechanics in left ventricular myocytes. (A,B) Representative examples of calcium transients (A: normalized traces) and sarcomere shortening (B) recorded from control (C, solid line), diabetic (D, dotted line) and SAHA treated diabetic cells (D + SAHA, dashed line). In bar graphs C-J: Mean values \pm SEM of the amplitude of calcium transient $(\mathrm{C}: \mathrm{f} / \mathrm{f} 0)$, time-to-peak of the calcium transient (D: TTP), time constant of the rate of intracellular $\mathrm{Ca}^{2+}$ clearing (E: tau), fraction of shortening (F: FS), maximal rate of shortening (G: $\left.-\mathrm{dL} / \mathrm{dt}_{\max }\right)$, maximal rate of re-lengthening $\left(\mathbf{H},+\mathrm{dL} / \mathrm{dt}_{\max }\right)$, time to $50 \%$ and $90 \%$ re-lengthening (I: RL50\%; J: RL90\%), measured in 45 C, 61 D, and 48 D + SAHA cardiomyocytes (CMs). ${ }^{*} p<0.05$ significant differences vs. C; \# $p<0.05$ significant differences vs. D. (GLM-ANOVA for repeated measurements). 


\subsection{Molecular Assays}

\subsubsection{Western Blot}

We performed Western blot experiments to understand the potential molecular changes responsible for the amelioration of cardiac contractility and calcium dynamics that we observed in diabetic CMs exposed to SAHA $(2.5 \mu \mathrm{mol} / \mathrm{L})$. The pro-acetlylation effect of SAHA treatment was confirmed in each animal by evaluating the relative increase in acetyl-tubulin expression compared to total tubulin (Figure 2A).
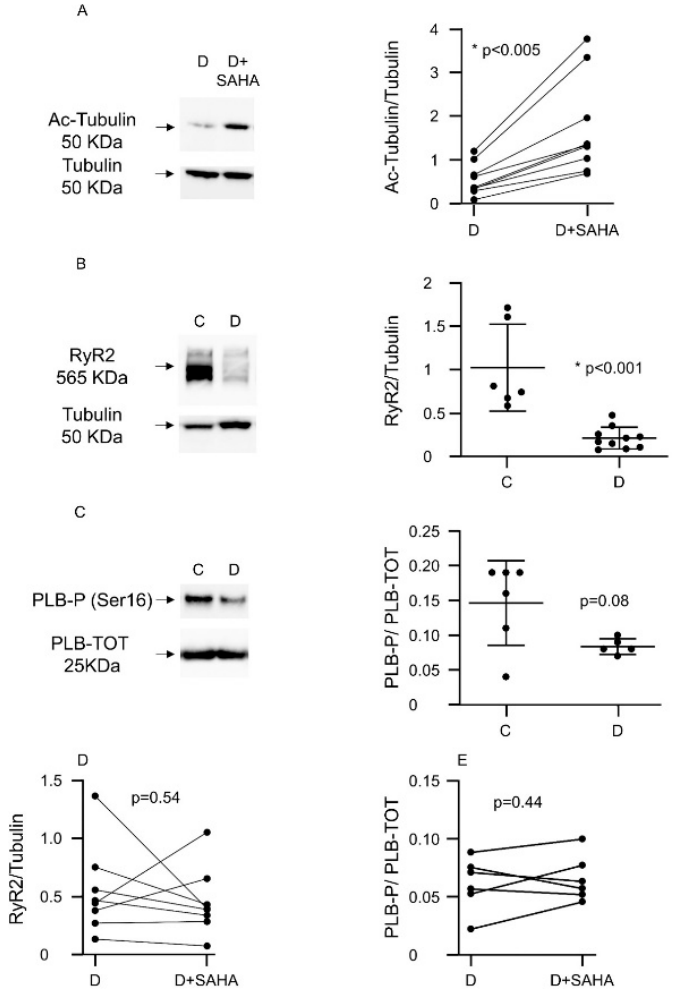

Figure 2. Effect of SAHA treatment on protein expression in left ventricular myocytes. (A) Western blot panels and densitometric analysis showing Ac-Tubulin protein expression after SAHA treatment in ventricular $\mathrm{CM}$ s isolated from nine $\mathrm{D}$ rats. Wilcoxon matched-pairs signed rank test: ${ }^{*} p<0.05$ vs. C; (B) Western blot panels and densitometric analysis showing RyR2 protein expression reduction in CMs of diabetic rats ( $n$. of rats $=10)$ compared to C cells $(n$. of rats $=6)$. Mann-Whitney U-test: $* p<0.001$ vs. C; (C) Western blot panels and densitometric analysis showing the expression of phosphorylated phospholamban (PLB-P, Ser16) compared to total phospholamban (PLB-TOT), in adult $\mathrm{C}$ cardiomyocytes vs. $\mathrm{D}$ cells $(n$. of $\mathrm{C}$ rats $=6 ; n$. of $\mathrm{D}$ rats $=5$ ), normalized to total protein concentration (Ponceau). Mann-Whitney U-test: $p=0.08$ vs. C; (D) Western blot panels and densitometric analysis showing RyR2 protein expression after SAHA treatment in diabetic CMs ( $n$. of rats $=8$ ). Wilcoxon matched-pairs signed rank test; (E) Western blot analysis showing the expression of phosphorylated phospholamban (PLB-P, Ser16) compared to total phospholamban (PLB-TOT) in adult rat CMs after SAHA treatment normalized to total protein concentration (Ponceau) ( $n$. of rats $=6$ ). Wilcoxon matched-pairs signed rank test.

In comparison with group $C$, untreated D cells exhibited a significant downregulation of ryanodine receptors (RyR2) (Figure 2B) and a decline of the ratio between the phosphorylated and the total form of phospholamban (PLB-P/PLB-TOT, $-45 \%$, Figure $2 \mathrm{C}$ ), although this last difference did not reach statistical significance $(p=0.08)$. SAHA treatment did not induce the recovery of RyR2 expression levels (Figure 2D) and P-PLB/PLB-TOT ratios (Figure 2E). At this stage of the diabetic disease, the expression levels of the Sarco/Endoplasmic Reticulum $\mathrm{Ca}^{2+}$-ATPase (SERCA), the voltage-dependent L-type 
calcium channel (subunit alpha, CACNA1c) and the $\mathrm{Na}^{+} / \mathrm{Ca}^{2+}$ exchanger (isoform $1, \mathrm{NCX} 1$ ) were unchanged, in both treated and untreated diabetic $\mathrm{CMs}$

\subsubsection{Cardiomyocyte ATP Content, Metabolic Activity, and Intracellular ROS Levels}

In order to explain, at least in part, the lower contractile efficiency and altered calcium dynamics in diabetic CMs, as well as the recovery of the cell mechanical properties induced by SAHA exposure, we measured the total intracellular ATP content. However, the ATP availability, primarily produced by the mitochondrial oxidative phosphorylation, was comparable in all groups, with only slight differences, independent of the treatment (Figure 3).

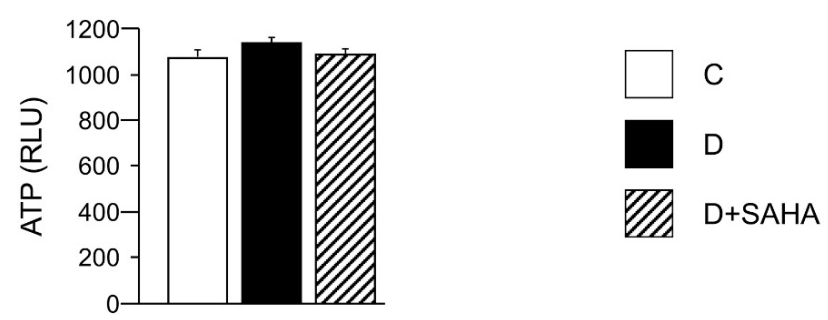

Figure 3. Effect of SAHA treatment on cardiomyocyte ATP content. Mean values \pm SEM of ATP content in control (C), diabetic (D), and SAHA-treated D cells (D + SAHA). Values are expressed as relative light units (RLU) counts/s. Kruskal-Wallis and U Mann-Whitney test.

Nevertheless, when the dehydrogenase activity was analyzed, a significant $(p<0.05)$ reduction was found in D cardiomyocytes in comparison with C cells, independent of SAHA treatment (Figure 4A,B). Conversely, the exposure of D cells to SAHA (D + SAHA) completely abolished the increase in intracellular ROS content observed in untreated D cardiomyocytes, restoring the values measured in $C$ cells (Figure 4C,D).
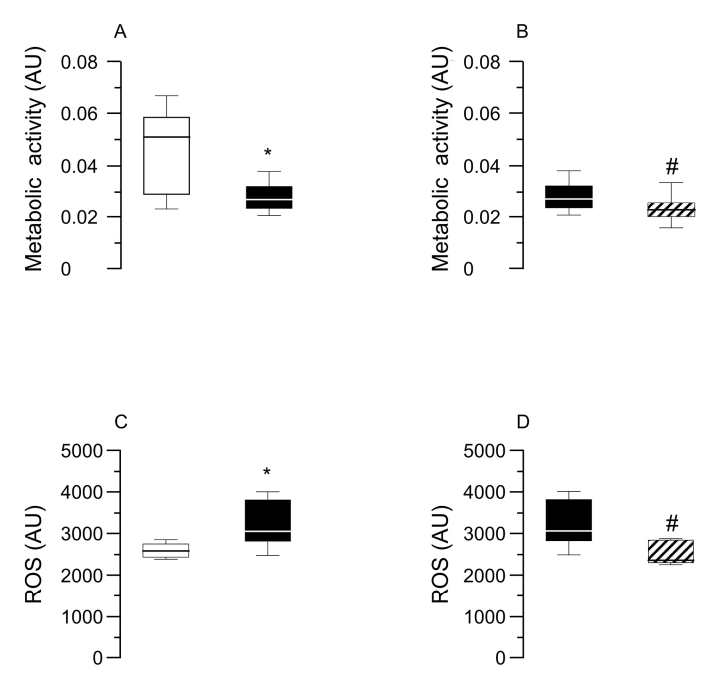

с $\square$ D $\mathscr{W} \mathrm{D}+\mathrm{SAHA}$

Figure 4. Effect of SAHA treatment on CMs' metabolic activity and intracellular ROS content. Data are reported as median values and interquartile range. In left panels: Effect of diabetes on CM metabolic activity (A) and ROS levels (C), compared with control cells. Mann-Whitney U-test: ${ }^{*} p<0.05$ vs. group C. In right panels: metabolic activity (B) and ROS content (D) measured in diabetic CMs before and after SAHA-exposure. Cells were isolated from the heart of three normal rats (group C) and five diabetic rats (groups D and D + SAHA). AU = arbitrary units. \# $p<0.05$ vs. D group; Wilcoxon matched-pairs signed rank test. 


\section{Discussion}

Diabetes and other metabolic conditions characterized by elevated blood glucose constitute important risk factors for cardiovascular disease. Hyperglycemia per se can directly target myocardial cells and the tissue microenvironment [4], progressively leading to ineffective electro-mechanical properties of the heart at advanced stages of diabetes [21]. Hence, the importance of identifying new adjuvant pharmacological approaches able to prevent or reduce the initial cellular alterations dictating the progressive deterioration of cardiac function. In this context, the main objective of the present study was to assess whether HDAC inhibition could reverse early abnormalities occurring in CMs after a short period of hyperglycemia.

Taken together, our results suggest that at the very early stages of the diabetic disease, SAHA-induced HDAC inhibition can counteract the occurrence of the first signs of CM dysfunction mainly by reducing intracellular ROS accumulation.

We used a model of STZ-induced early diabetes widely characterized from a morpho-functional point-of-view. We confirmed that three weeks of hyperglycemia corresponds to a transitional phase preceding the overt diabetic cardiomyopathy phenotype [4,24]. At this time point, an initial deterioration of the intrinsic contractile properties of CMs associated with altered intracellular calcium dynamics occurred in D cells compared with those of group $\mathrm{C}$. The slow $\mathrm{Ca}^{2+}$ transient decay and the parallel decrease in the rate of cell re-lengthening is indicative of either impaired sequestration of $\mathrm{Ca}^{2+}$ in the SR or a reduced calcium extrusion through the plasmalemma sodium/calcium exchanger (NCX), the two most important players involved in cytosolic calcium clearing [25,26]. At the molecular level, however, no substantial changes were detected in the expression levels of most proteins involved in the excitation-contraction process, namely SERCA2, NCX, and L-type calcium channels. Conversely, a significant down-regulation of RyR2 expression was observed in untreated D cells that may explain the delayed time-to-peak of the $\mathrm{Ca}^{2+}$ transient. This finding confirms that a decrease in RyR2 expression level, already known to occur in more advanced phases of diabetes [27], may represent an important early component of the perturbed calcium cycling of hyperglycemic myocytes [21]. The reactive oxygen species accumulation observed in D CMs can also contribute to RyR2 dysfunction by altering their redox state [28] and phosphorylation [29]. In addition, we recently showed that, at these stages of diabetes, the oxidative stress promotes a cellular inflammatory response, implying the release of Monocyte Chemoattractant protein 1 (MCP-1) and Fractalkine pro-inflammatory chemokines from parenchymal and stromal cardiac cell compartments [4]. It should be outlined that Fractalkine, besides its chemo-attractant action, can exert direct effects on the intracellular contractile machinery by binding to its receptor on the membrane of ventricular CMs [30,31]. ROS accumulation is also linked to alterations in mitochondrial function and can have noxious effects by directly acting on different redox-sensitive target molecules involved in the signal-transduction pattern, as well as proteins that participate in the excitation-contraction process, including SERCA2 whose increased oxidation results in contractile alterations [29].

Although other mechanisms cannot be excluded, these findings support the hypothesis that the positive effects induced by low-dose SAHA treatment on cardiomyocyte performance can be attributed, at least in part, to its capacity of reducing ROS accumulation, an early event in the diabetic heart following diabetes-induced metabolic changes. This interpretation is also supported by previous reports showing that scavenging of ROS improves calcium homeostasis and attenuates cardiac derangement in multiple models of heart disease, including heart failure, hypertrophy, diabetic cardiomyopathy, and aging $[16,32,33]$. The difference in metabolic activity between control and diabetic rats, revealed through MTS assay, could be related to an impairment of mitochondrial function induced by the oxidative stress, in particular the loss of mtDNA integrity that can have consequences on bioenergetics and metabolism [34]. A concomitant decrease in metabolic processes, mainly $\mathrm{NAD}(\mathrm{P}) \mathrm{H}$ dehydrogenase activities and ROS levels, was found in diabetic CMs after SAHA exposure, suggesting a possible mechanistic correlation among cell metabolism and altered ROS production. It could be 
hypothesized that SAHA helps in restoring a correct redox signaling in diabetic cells, which is essential for the maintenance of cardiomyocyte homeostasis and is involved in the cardiac response to stress [35].

In conclusion, we provided here a proof of concept that the modulation of HAT/HDAC activity can counteract early diabetes-induced oxidative stress and functional changes in CMs. Such observations constitute the starting point for further mechanistic studies aimed at identifying new adjuvant pharmacological approaches capable of preventing the initial myocardial damage occurring in the diabetic heart and its progression towards an overt, clinically relevant, pathological condition.

\section{Materials and Methods}

The investigation was approved by the Veterinary Animal Care and Use Committee of the University of Parma-Italy (31 March 2016) and conforms to the National Ethical Guidelines of the Italian Ministry of Health (Prot. N ${ }^{\circ}$ 614/2016-PR; 17 June 2016). Euthanasia in experimental animals was performed under ketamine chloride anesthesia (Imalgene, Merial, Milan, Italy; $40 \mathrm{mg} / \mathrm{kg}$ i.p.) plus medetomidine hydrochloride (Domitor, Pfizer Italia S.r.l., Latina, Italy; $0.15 \mathrm{mg} / \mathrm{kg}$ i.p). The details of the experimental procedures reported under Sections $4.2-4.5$ have been already described $[23,24,36]$ (see also in the Supplementary Material).

\subsection{Animals and Experimental Study}

The study population consisted of 11 male Wistar rats (Rattus norvegicus) aged 12-14 weeks, weighing $394 \pm 8 \mathrm{~g}$. Five animals were used as the control group (C group) while in six animals (D group), diabetes was induced by a single intra-peritoneal injection of streptozotocin (STZ, $60 \mathrm{mg} / \mathrm{kg})$. All animals had normal blood glucose levels at the beginning of the experimental protocol $(106 \pm 3 \mathrm{mg} / \mathrm{dL})$. Two days after STZ injection, glycaemia significantly rose in D rats compared with $C(360 \pm 50 \mathrm{mg} / \mathrm{dL}$ vs. $107 \pm 4 \mathrm{mg} / \mathrm{dL} ; p<0.01$ ). During the subsequent weeks, blood glucose levels remained stable in both $C$ and $D$ groups until the end of the experimental protocol (after three weeks of hyperglycemia: $\mathrm{C}=103 \pm 1 \mathrm{mg} / \mathrm{dL} ; \mathrm{D}: 375 \pm 24 \mathrm{mg} / \mathrm{dL}$ ). A $5 \%$ decrease in body weight was observed in diabetic animals during the first two weeks after STZ injection. Subsequently, body mass exhibited only negligible changes while in C animals it slightly increased (body weight at sacrifice: $C=423 \pm 12 \mathrm{~g}$; D: $374 \pm 20 \mathrm{~g}$ ). Three weeks after the STZ or vehicle injection, the animals were anesthetized and the heart was rapidly removed after decapitation. Ventricular CMs were enzymatically isolated for the ex-vivo measure of the functional parameters and then stored at $-80^{\circ} \mathrm{C}$ for subsequent molecular assay (see Section 4.4).

Twelve additional age-matched animals (7 C and 5 D) were used to increase the sample size for molecular assay and to evaluate the cardiomyocyte ATP content, metabolic activity, and ROS levels (see Sections 4.4-4.7).

\subsection{Myocyte Isolation}

From the heart of each animal, individual ventricular myocytes were enzymatically isolated by collagenase perfusion in accordance with a procedure previously described [24]. Then, cells were used for measuring sarcomere shortening and calcium transients (IonOptix, Milton, MA, USA). Functional measurements in CMs isolated from diabetic hearts were performed before (D group) and after exposure to SAHA $(2.5 \mu \mathrm{mol} / \mathrm{L})$ for $90 \mathrm{~min}$ (D + SAHA) [23]. The concentration and the period of SAHA exposure have been selected on the basis of previous in vitro/ex-vivo studies by our group [23] and other investigators [37]. It has been shown that SAHA in the range of 1 to $5 \mu \mathrm{mol} / \mathrm{L}$ increases histone acetylation in an in vitro model within one hour, reaching the plateau effect at $3 \mathrm{~h} \mathrm{[35].} \mathrm{We} \mathrm{also}$ recently reported that $90 \mathrm{~min}$ are sufficient to achieve the acetylation increase of other HDAC potential targets, including cytoplasmic substrates, and to preserve cell viability [23]. 


\subsection{Cardiomyocyte Mechanics and Calcium Transients}

Cardiomyocyte contractile properties and calcium dynamics were simultaneously recorded from a total of 45 control, 61 D, and 48 D + SAHA isolated LV myocytes in order to measure the following parameters: the mean diastolic sarcomere length and the fraction of shortening (FS); the maximal rates of shortening and re-lengthening $\left( \pm \mathrm{dL} / \mathrm{dt}_{\max }\right)$; the time at $10 \%, 50 \%$, and $90 \%$ of re-lengthening (RL10\%, RL50\% and RL90\%, respectively), the amplitude of the calcium transient (expressed as normalized fluorescence (f/f0); the time-to-peak of the calcium transients (TTP) and the time constant (tau) of the fluorescence signal decay, taken as an index of the rate of intracellular calcium clearing [25].

\subsection{Western Blot Analysis}

SDS-PAGE and immunoblot were performed as previously described [23]. The following primary antibodies were used SERCA2 (1:1000, SantaCruz Biotecnology, Dallas, TX, USA, sc-376235), Acetylated-tubulin (1:1000, Sigma-Aldrich, St. Louis, MO, USA \# T7451), Tubulin (1:2000, Abcam, Cambridge, UK, \# ab59680), Ryanodine Receptor (C3-33) (1:1000, Abcam \# ab2827), CACNA1c (1:500, Abcam \# ab81095), NCX-1 (1:250, SantaCruz Biotecnology, Dallas, TX, USA \#sc-32881), Phospho-Phospholamban (Ser16) (1:5000, Merck Millipore, Burlington, MA, USA \# 07-052), Phospholamban (1:8000, Abcam \#ab2865). The band density was evaluated using the Image Lab software 5.2.1 (BioRad, Hercules, CA, USA) and quantifications were normalized either to total protein concentration (Ponceau red staining) or Tubulin.

\subsection{ATP Content in Left Ventricular Myocytes}

Frozen CMs isolated from two C and four D rats, either untreated or exposed to SAHA for $90 \mathrm{~min}$, were resuspended in phosphate buffered solution (PBS) and aliquoted in triplicate in a 96-well white plate, as previously described [36]. The ATP intracellular content was measured by the Luminescence ATP Detection Assay System (ATPlite) (PerkinElmer, Waltham, MA, USA) according to the manufacturer's instructions. Briefly, cells were lysed, shaken, and dark incubated with the substrate solution. Then, the luminescence intensity was measured by the EnSpire ${ }^{\circledR}$ multimode plate reader (PerkinElmer) and normalized for the total protein content of each sample.

\subsection{Cardiomyocyte Metabolic Activity}

Metabolic activity was detected through CellTiter96R AQueous One Solution Cell Proliferation Assay (MTS) (Promega Corporation, Madison, WI, USA) in ventricular CMs isolated from three control and five diabetic rats. Aliquots of isolated D CMs $(1.5 \mathrm{~mL})$, at a final concentration of approximately $12 \times 10^{4} \mathrm{cell} / \mathrm{mL}$, were either untreated or exposed to SAHA $(2.5 \mu \mathrm{mol} / \mathrm{L})$ for $90 \mathrm{~min}$. Afterwards, cells were re-suspended in fresh medium and $100 \mu \mathrm{L}$ of cell suspension and were seeded, in triplicate, in a 96-well plate. Twenty microliters of the CellTiter96R AQ One Solution Cell Proliferation Assay were added to each well; cells were incubated at $37^{\circ} \mathrm{C}$ in a humidified (95\%), $\mathrm{CO}_{2}(5 \%)$ incubator for $4 \mathrm{~h}$, and then the absorbance at $485 \mathrm{~nm}$ was recorded with a 96-well plate reader (TECAN SpectraFluor Plus, Männedorf, Switzerland). Differences in absorbance were correlated to different activities of the dehydrogenases.

\subsection{ROS Assay}

ROS assays were performed on aliquots of ventricular CMs isolated from the same five D rats used for detecting metabolic activity. To measure cytoplasmic ROS levels, $6 \mathrm{~mL}$ of CMs, at a final concentration of approximately $12 \times 10^{4}$ cell $/ \mathrm{mL}$, were incubated for $30 \mathrm{~min}$ at $37{ }^{\circ} \mathrm{C}$ with medium containing DCFH-DA (33 $\mu \mathrm{mol} / \mathrm{L})$ and subsequently washed twice with PBS. SAHA-treated and untreated D cells were transferred, in triplicate, in a 96-well black plate. Fluorescence signals were recorded using a plate reader (TECAN SpectraFluor Plus, Männedorf, Switzerland) (excitation 485 nm; emission $535 \mathrm{~nm}$ ). A positive control was performed treating cells with $0.003 \% \mathrm{H}_{2} \mathrm{O}_{2}$ for 20 min. 


\subsection{Statistics}

Normality of the data was evaluated by the D'Agostino and Pearson normality test. The results are reported as means \pm standard error (SEM) or median and interquartile range for variables that were not normally distributed. The statistical treatment of data includes: General Linear Model (GLM) ANOVA for repeated measurements, Kruskal-Wallis, and U Mann-Whitney non-parametric statistical tests. For two groups, significance was assessed by Mann-Whitney U-test or Wilcoxon matched-pairs signed rank test, when appropriate (IBM-SPSS 24.0, SPSS Inc., Chicago, IL, USA). The specific test used is reported in the figure legend of each experiment. A $p$-value $<0.05$ was considered statistically significant.

Supplementary Materials: Supplementary materials can be found at http://www.mdpi.com/1422-0067/20/8/1873/ s1.

Author Contributions: Conceptualization, D.S. and A.R.; Methodology, D.S., A.R., F.R., A.B.; investigation (cardiomyocyte function) L.B., M.S., R.V.; investigation (molecular data) B.M.M., V.M., S.G., R.V., M.L.; formal analysis, L.B., M.S., R.V., B.M.M., V.M., S.G., R.V., M.L.; visualization, L.B., B.M.M.; supervision, D.S., A.R., F.R., A.B., P.P.P.; writing—original draft preparation, D.S., A.R.; writing-review and editing, D.S., A.R., F.R., A.B.

Funding: This work was supported by the Department of Innovation, Research and Universities of the Autonomous Province of Bolzano-South Tyrol (Italy) and by local research funding from Parma University (FIL2016).

Acknowledgments: The authors would like to thank Andrew Hicks for English revision.

Conflicts of Interest: The authors declare no conflict of interest. The funders had no role in the design of the study; in the collection, analyses, or interpretation of data; in the writing of the manuscript, or in the decision to publish the results.

\section{References}

1. Hölscher, M.E.; Bode, C.; Bugger, H. Diabetic Cardiomyopathy: Does the Type of Diabetes Matter? Int. J. Mol. Sci. 2016, 17, 2136. [CrossRef] [PubMed]

2. Zhang, L.; Du, J.; Yano, N.; Wang, H.; Zhao, Y.T.; Dubielecka-Szczerba, P.; Zhuang, S.; Chin, E.Y.; Qin, G.; Zhao, T.C. Sodium butyrate protects against high fat diet-induced cardiac dysfunction and metabolic disorders in type II diabetic mice. J. Cell Biochem. 2017, 118, 2395-2408. [CrossRef] [PubMed]

3. Jia, G.; Whaley-Connell, A.; Sowers, J.R. Diabetic cardiomyopathy: A hyperglycaemia- and insulin-resistanceinduced heart disease. Diabetologia 2018, 61, 21-28. [CrossRef] [PubMed]

4. Savi, M.; Bocchi, L.; Sala, R.; Frati, C.; Lagrasta, C.; Madeddu, D.; Falco, A.; Pollino, S.; Bresciani, L.; Miragoli, M.; et al. Parenchymal and Stromal Cells Contribute to Pro-Inflammatory Myocardial Environment at Early Stages of Diabetes: Protective Role of Resveratrol. Nutrients 2016, 8, 729. [CrossRef]

5. Bugger, H.; Abel, E.D. Molecular mechanisms of diabetic cardiomyopathy. Diabetologia 2014, 57, 660-671. [CrossRef] [PubMed]

6. Zhao, Y.; Zhang, L.; Qiao, Y.; Zhou, X.; Wu, G.; Wang, L.; Peng, Y.; Dong, X.; Huang, H.; Si, L.; et al. Heme oxygenase-1 prevents cardiac dysfunction in streptozotocin-diabetic mice by reducing inflammation, oxidative stress, apoptosis and enhancing autophagy. PLOS ONE 2013, 8, e75927. [CrossRef] [PubMed]

7. Delucchi, F.; Berni, R.; Frati, C.; Cavalli, S.; Graiani, G.; Sala, R.; Chaponnier, C.; Gabbiani, G.; Calani, L.; Del Rio, D.; et al. Resveratrol treatment reduces cardiac progenitor cell dysfunction and prevents morpho-functional ventricular remodeling in type-1 diabetic rats. PLoS ONE 2012, 7, e39836. [CrossRef]

8. Rajesh, M.; Mukhopadhyay, P.; Bátkai, S.; Patel, V.; Saito, K.; Matsumoto, S.; Kashiwaya, Y.; Horváth, B.; Mukhopadhyay, B.; Becker, L.; et al. Cannabidiol attenuates cardiac dysfunction, oxidative stress, fibrosis, and inflammatory and cell death signaling pathways in diabetic cardiomyopathy. J. Am. Coll. Cardiol. 2010, 56, 2115-2125. [CrossRef]

9. Stilli, D.; Lagrasta, C.; Berni, R.; Bocchi, L.; Savi, M.; Delucchi, F.; Graiani, G.; Monica, M.; Maestri, R.; Baruffi, S.; et al. Preservation of ventricular performance at early stages of diabetic cardiomyopathy involves changes in myocyte size, number and intercellular coupling. Basic Res. Cardiol. 2007, 102, 488-499. [CrossRef]

10. Frati, G.; Schirone, L.; Chimenti, I.; Yee, D.; Biondi-Zoccai, G.; Volpe, M.; Sciarretta, S. An overview of the inflammatory signalling mechanisms in the myocardium underlying the development of diabetic cardiomyopathy. Cardiovasc. Res. 2017, 113, 378-388. [CrossRef] 
11. Khan, S.; Ahirwar, K.; Jena, G. Anti-fibrotic effects of valproic acid: Role of HDAC inhibition and associated mechanisms. Epigenomics 2016, 8, 1087-1101. [CrossRef]

12. Khan, S.; Kowluru, A. CD36 mediates lipid accumulation in pancreatic beta cells under the duress of glucolipotoxic conditions: Novel roles of lysine deacetylases. Biochem. Biophys. Res. Commun. 2018, 495, 2221-2226. [CrossRef]

13. Ferguson, B.S.; McKinsey, T.A. Non-sirtuin histone deacetylases in the control of cardiac aging. J. Mol. Cell Cardiol. 2015, 83, 14-20. [CrossRef]

14. Gallo, P.; Latronico, M.V.G.; Gallo, P.; Grimaldi, S.; Borgia, F.; Todaro, M.; Jones, P.; Gallinari, P.; De Francesco, R.; Ciliberto, G.; et al. Inhibition of class I histone deacetylase with an apicidin derivative prevents cardiac hypertrophy and failure. Cardiovasc. Res. 2008, 80, 416-424. [CrossRef]

15. Grant, S.; Easley, C.; Kirkpatrick, P. Vorinostat. Nat. Rev. Drug Discov. 2007, 6, 21-22. [CrossRef]

16. Chen, Y.; Du, J.; Zhao, Y.T.; Zhang, L.; Lv, G.; Zhuang, S.; Qin, G.; Zhao, T.C. Histone deacetylase (HDAC) inhibition improves myocardial function and prevents cardiac remodeling in diabetic mice. Cardiovasc. Diabetol. 2015, 14, 99. [CrossRef]

17. Manea, S.A.; Antonescu, M.L.; Fenyo, I.M.; Raicu, M.; Simionescu, M.; Manea, A. Epigenetic regulation of vascular NADPH oxidase expression and reactive oxygen species production by histone deacetylase-dependent mechanisms in experimental diabetes. Redox Biol. 2018, 16, 332-343. [CrossRef]

18. Xu, Z.; Tong, Q.; Zhang, Z.; Wang, S.; Zheng, Y.; Liu, Q.; Qian, L.B.; Chen, S.Y.; Sun, J.; Cai, L. Inhibition of HDAC3 prevents diabetic cardiomyopathy in OVE26 mice via epigenetic regulation of DUSP5-ERK1/2 pathway. Clin. Sci. (Lond.) 2017, 131, 1841-1857. [CrossRef]

19. Wu, Y.; Leng, Y.; Meng, Q.; Xue, R.; Zhao, B.; Zhan, L.; Xia, Z. Suppression of Excessive Histone Deacetylases Activity in Diabetic Hearts Attenuates Myocardial Ischemia/Reperfusion Injury via Mitochondria Apoptosis Pathway. J. Diabetes Res. 2017, 8208065. [CrossRef]

20. Sulaiman, M.; Matta, M.J.; Sunderesan, N.R.; Gupta, M.P.; Periasamy, M.; Gupta, M. Resveratrol, an activator of SIRT1, upregulates sarcoplasmic calcium ATPase and improves cardiac function in diabetic cardiomyopathy. Am. J. Physiol. Heart Circ. Physiol. 2010, 298, H833-H843. [CrossRef]

21. Sorrentino, A.; Borghetti, G.; Zhou, Y.; Cannata, A.; Meo, M.; Signore, S.; Anversa, P.; Leri, A.; Goichberg, P.; Qanud, K.; et al. Hyperglycemia induces defective $\mathrm{Ca}^{2+}$ homeostasis in cardiomyocytes. Am. J. Physiol. Heart Circ. Physiol. 2017, 312, H150-H161. [CrossRef]

22. Butler, L.M.; Zhou, X.; Xu, W.S.; Scher, H.I.; Rifkind, R.A.; Marks, P.A.; Richon, V.M. The histone deacetylase inhibitor SAHA arrests cancer cell growth, up-regulates thioredoxin-binding protein-2, and down-regulates thioredoxin. Proc. Natl. Acad. Sci. USA 2002, 99, 11700-11705. [CrossRef] [PubMed]

23. Meraviglia, V.; Bocchi, L.; Sacchetto, R.; Florio, M.C.; Motta, B.M.; Corti, C.; Weichenberger, C.X.; Savi, M.; D'Elia, Y.; Rosato-Siri, M.D.; et al. HDAC Inhibition Improves the Sarcoendoplasmic Reticulum Ca ${ }^{2+}$-ATPase Activity in Cardiac Myocytes. Int. J. Mol. Sci. 2018, 19, 419. [CrossRef]

24. Savi, M.; Bocchi, L.; Mena, P.; Dall'Asta, M.; Crozier, A.; Brighenti, F.; Stilli, D.; Del Rio, D. In vivo administration of urolithin A and B prevents the occurrence of cardiac dysfunction in streptozotocin induced diabetic rats. Cardiovasc. Diabetol. 2017, 16, 80. [CrossRef] [PubMed]

25. Bassani, J.W.; Bassani, R.A.; Bers, D.M. Relaxation in rabbit and rat cardiac cells: Species dependent differences in cellular mechanisms. J. Physiol. 1994, 476, 279-293. [CrossRef]

26. Eisner, D.A.; Caldwell, J.L.; Kistamás, K.; Trafford, A.W. Calcium and Excitation-Contraction Coupling in the Heart. Circ. Res. 2017, 121, 181-195. [CrossRef]

27. Wang, M.; Zhang, W.B.; Zhu, J.H.; Fu, G.S.; Zhou, B.Q. Breviscapine ameliorates cardiac dysfunction and regulates the myocardial $\mathrm{Ca}^{2+}$-cycling proteins in streptozotocin-induced diabetic rats. Acta Diabetol. 2010, 209-218. [CrossRef]

28. Nikolaienko, R.; Bovo, E.; Zima, A.V. Redox Dependent Modifications of Ryanodine Receptor: Basic Mechanisms and Implications in Heart Diseases. Front. Physiol. 2018, 9, 1775. [CrossRef]

29. Burgoyne, J.R.; Mongue-Din, H.; Eaton, P.; Shah, A.M. Redox signaling in cardiac physiology and pathology. Circ. Res. 2012, 111, 1091-1106. [CrossRef]

30. Taube, D.; Xu, J.; Yang, X.P.; Undrovinas, A.; Peterson, E.; Harding, P. Fractalkine depresses cardiomyocyte contractility. PLoS ONE 2013, 8, e69832. [CrossRef] 
31. Husberg, C.; Nygård, S.; Finsen, A.V.; Damås, J.K.; Frigessi, A.; Øie, E.; Wæhre, A.; Gullestad, L.; Aukrust, P.; Yndestad, A.; et al. Cytokine expression profiling of the myocardium reveals a role for CX3CL1 (fractalkine) in heart failure. J. Mol. Cell. Cardiol. 2008, 45, 261-269. [CrossRef]

32. Jin, L.; Piao, Z.H.; Liu, C.P.; Sun, S.; Liu, B.; Kim, G.R.; Choi, S.Y.; Ryu, Y.; Kee, H.J.; Jeong, M.H. Gallic acid attenuates calcium calmodulin-dependent kinase II-induced apoptosis in spontaneously hypertensive rats. J. Cell. Mol. Med. 2018, 22, 1517-1526. [CrossRef]

33. Chacar, S.; Hajal, J.; Saliba, Y.; Bois, P.; Louka, N.; Maroun, R.G.; Faivre, J.F.; Fares, N. Long-term intake of phenolic compounds attenuates age-related cardiac remodeling. Aging Cell. 2019, e12894. [CrossRef]

34. McLaughlin, K.L.; McClung, J.M.; Fisher-Wellman, K.H. Bioenergetic consequences of compromised mitochondrial repair in the mouse heart. Biochem. Biophys. Res. Commun. 2018, 504, 742-748. [CrossRef]

35. Santos, C.X.; Anilkumar, N.; Zhang, M.; Brewer, A.C.; Shah, A.M. Redox signaling in cardiac myocytes. Free Radic. Biol. Med. 2011, 50, 777-793. [CrossRef]

36. Bocchi, L.; Savi, M.; Naponelli, V.; Vilella, R.; Sgarbi, G.; Baracca, A.; Solaini, G.; Bettuzzi, S.; Rizzi, F.; Stilli, D. Long-Term Oral Administration of Theaphenon-E Improves Cardiomyocyte Mechanics and Calcium Dynamics by Affecting Phospholamban Phosphorylation and ATP Production. Cell. Physiol. Biochem. 2018, 47, 1230-1243. [CrossRef]

37. Tiffon, C.; Adams, J.; van der Fits, L.; Wen, S.; Townsend, P.; Ganesan, A.; Hodges, E.; Vermeer, M.; Packham, G. The histone deacetylase inhibitors vorinostat and romidepsin downmodulate IL-10 expression in cutaneous T-cell lymphoma cells. Br. J. Pharmacol. 2011, 162, 1590-1602. [CrossRef]

(C) 2019 by the authors. Licensee MDPI, Basel, Switzerland. This article is an open access article distributed under the terms and conditions of the Creative Commons Attribution (CC BY) license (http://creativecommons.org/licenses/by/4.0/). 